\title{
Imaging Biomarkers in Acute Ischemic Stroke Trials: A Systematic Review
}

\author{
G.W.J. Harston, N. Rane, G. Shaya, S. Thandeswaran, M. Cellerini, F. Sheerin, and J. Kennedy
}

\section{ABSTRACT}

BACKGROUND AND PURPOSE: Imaging biomarkers are increasingly used to provide a better understanding of the pathophysiology of acute ischemic stroke. However, this approach of routinely using imaging biomarkers to inform treatment decisions has yet to be translated into successful randomized trials. The aim of this study was to systematically review the use of imaging biomarkers in randomized controlled trials in patients with acute ischemic stroke, exploring the purposes for which the imaging biomarkers were used.

MATERIALS AND METHODS: We performed a systematic review of imaging biomarkers used in randomized controlled trials of acute ischemic stroke, in which a therapeutic intervention was trialed within 48 hours of symptom onset. Data bases searched included MEDLINE, EMBASE, strokecenter.org, and the Virtual International Stroke Trials Archive (1995-2014).

RESULTS: Eighty-four studies met the criteria, of which 49 used imaging to select patients; 31, for subgroup analysis; and 49, as an outcome measure. Imaging biomarkers were broadly used for 8 purposes. There was marked heterogeneity in the definitions and uses of imaging biomarkers and significant publication bias among post hoc analyses.

CONCLUSIONS: Imaging biomarkers offer the opportunity to refine the trial cohort by minimizing participant variation, to decrease sample size, and to personalize treatment approaches for those who stand to benefit most. However, within imaging modalities, there has been little consistency between stroke trials. Greater effort to prospectively use consistent imaging biomarkers should help improve the development of novel treatment strategies in acute stroke and improve comparison between studies.

ABBREVIATIONS: $\mathrm{RCT}=$ randomized controlled trial; $\mathrm{TCD}=$ transcranial Doppler

$\mathbf{T}$ reatment options for patients with acute ischemic stroke are limited. Despite attempts to develop novel neuroprotectants and strategies for reperfusion, very few have made it into routine practice. This failure of progress is multifactorial in origin but includes failure to properly account for patient heterogeneity and a lack of proved surrogate outcomes. ${ }^{1}$

Imaging has been widely embraced, both in clinical practice and

Received August 4, 2014; accepted after revision October 24

From the Radcliffe Department of Medicine (G.W.J.H., G.S., S.T., J.K.), University of Oxford, Oxford, United Kingdom; Department of Neuroradiology (N.R., M.C., F.S.), Oxford University Hospitals NHS Trust, Oxford, United Kingdom; and Neurointervention Service (N.R.), Royal Melbourne Hospital, Melbourne Health, Victoria, Australia.

This work was supported by the National Institute for Health Research Oxford Biomedical Research Centre Programme, the Dunhill Medical Trust (grant number OSRP1/1006), and the Centre of Excellence for Personalized Healthcare funded by the Wellcome Trust and Engineering and Physical Sciences Research Council (grant number WT 088877/Z/09/Z)

Preliminary analysis of some of these data previously presented in poster form at: European Stroke Conference, May 28-31, 2013; London, UK; and World Federation of Interventional and Therapeutic Neuroradiology, November 9-13, 2013; Buenos Aires, Argentina. research studies, to achieve various aims, including the following: reducing the heterogeneity of participants in a trial; stratifying patients into those who may or may not benefit from treatments; and assessing intervention efficacy and/or safety. ${ }^{2,3}$ The Acute Stroke Imaging Research Roadmap II was developed on the background of recent null acute stroke trials incorporating selection by using penumbral imaging. ${ }^{2}$ It reinforced the need for a rigorous definition of regions of interest, defined a framework for using imaging biomarkers in imaging studies (with the specific example of revascularization), and called for coordinated imaging data collection.

The aim of this study was to systematically review randomized intervention trials in patients with acute ischemic stroke, using the groupings developed in the Acute Stroke Imaging Research Roadmap II and detailing the purposes for which they were used.

Please address correspondence to George Harston, AVIC, University of Oxford, Level 2, John Radcliffe Hospital, Headington, Oxford, OX3 9DU, UK; e-mail: george. harston@rdm.ox.ac.uk; @AMIClstroke

- Indicates open access to non-subscribers at www.ajnr.org

= Indicates article with supplemental on-line appendix and tables.

http://dx.doi.org/10.3174/ajnr.A4208 


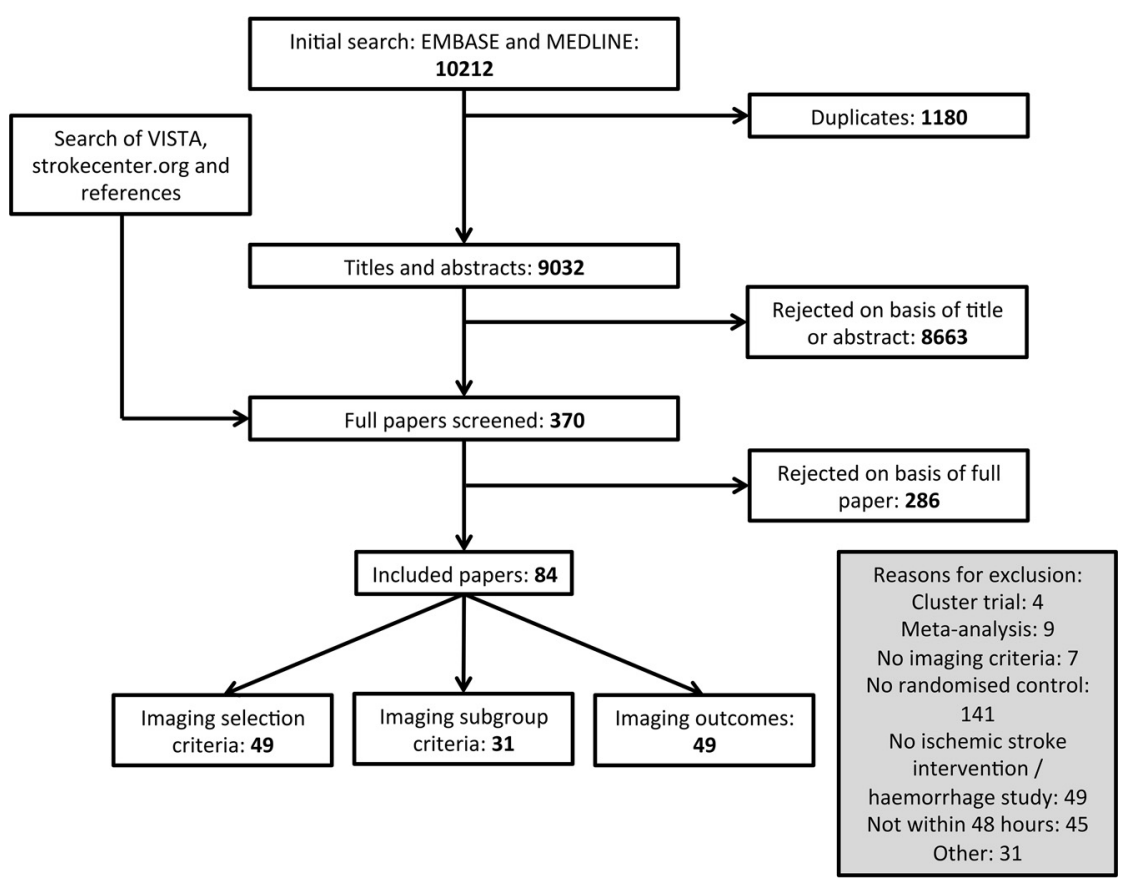

FIG 1. Schema of systematic review.

Table 1: Use of imaging in stroke trials

\begin{tabular}{lccc}
\hline & $\begin{array}{c}\text { Trial } \\
\text { Eligibility }\end{array}$ & $\begin{array}{c}\text { Outcome } \\
\text { Measure }\end{array}$ & $\begin{array}{c}\text { Subgroup } \\
\text { Analysis }\end{array}$ \\
\hline Infarct volume & 29 & 62 & 34 \\
Edema & 5 & NA & 0 \\
Diagnosis/territory of infarction & 9 & NA & 5 \\
Vessel status & 16 & 17 & 23 \\
Ischemic penumbra & 10 & 7 & 22 \\
Perfusion status only & 2 & 27 & 1 \\
Collaterals & 0 & 0 & 2 \\
Composite/other & 0 & 4 & 2 \\
\hline
\end{tabular}

Note:-NA indicates not applicable.

\section{MATERIALS AND METHODS Eligibility Criteria}

This systematic review selected studies analyzing data from randomized controlled trials (RCTs) of acute ischemic stroke therapies (eg, intravenous thrombolysis, glycemic control, and hypothermia). Subject enrollment was required within 48 hours of symptom onset, and imaging biomarkers were used to select patients, measure outcome, or define subgroups (preplanned or post hoc). Any form of imaging including CT, MRI, SPECT, or transcranial Doppler (TCD) was allowed. Studies that used imaging solely to exclude patients with intracranial hemorrhage at trial enrollment were not included in the analysis. Other exclusion criteria were the following: studies of hemorrhagic stroke or transient ischemic attack, cluster trials, studies in children, those not comparing treatment and control groups, and those using historical controls. When $\geq 2$ articles described the same populations, both studies were included, provided different imaging biomarkers were used in each.

\section{Search Strategy}

MEDLINE and EMBASE (1995 to March 2014) were searched by using a combination of terms, their derivatives, and related terms: RCTs, acute stroke, and imaging (On-line Appendix). Searches were limited to English language articles with adult human subjects. Additional searches were made of strokecenter.org and the Virtual International Stroke Trials Archive (www.vista.gla.ac.uk). Two of the 3 reviewers screened each title and abstract independently (G.W.J.H., N.R., G.S.). The same reviewers independently reviewed the full texts of all potentially relevant studies, and those included were accepted by consensus (Fig 1).

\section{Data Extraction}

Data extracted included year, number of patients enrolled, details of the imaging biomarker, and the results of the trial. The imaging biomarkers were classified into the groups identified in the Acute Stroke Imaging Research Roadmap II with particular attention to TreatmentRelevant Acute Imaging Targets. ${ }^{2}$ The purpose for which each group was used (trial eligibility, outcome measure, or subgroup analyses [preplanned or post hoc]) was recorded.

\section{RESULTS \\ Search Results}

The electronic search yielded 10,212 titles, of which 1180 were duplicates. Screening of the remaining studies and additional manual searching produced 370 to be appraised. Two hundred eighty-six were excluded following review of the article, leaving 84 to be included in the final analysis (Fig 1, On-line References). We identified 8 groups of imaging biomarkers: infarct volume, edema, diagnosis/territory of infarction, vessel status, ischemic penumbra, perfusion status, collaterals, and composite/other (Table 1). ${ }^{2}$ Forty-nine studies used imaging for trial eligibility, 49 studies used $\geq 1$ imaging outcome (eg, infarct volume or recanalization), and 31 studies used imaging criteria to define $\geq 1$ subgroup (eg, stratification by infarct volume). Eighty-five different imaging-defined subgroups were identified in these 31 studies, 17 of which were preplanned and the remaining 68 subgroups were defined post hoc.

\section{Imaging Biomarkers}

Infarct volume was extensively used as an exclusion criterion from trial recruitment and subgroup eligibility assessment (Table 1). The most consistent definition used was an infarct volume on noncontrast CT of greater than one-third of the MCA territory (16/29 studies) (On-line Table 1). Other definitions used ranged from any evidence of ischemia on noncontrast CT to greater than two-thirds of the MCA territory demonstrating restricted diffusion on DWI. One trial of hemicraniectomy for malignant MCA syndrome excluded patients with an infarct volume of less than a predefined level (145 mL on DWI). ${ }^{4}$

Imaging was used in a variety of other ways to establish trial 
Table 2: Proportion of analyses showing a positive outcome using imaging biomarkers for inclusion/exclusion or outcome assessment

\begin{tabular}{lccc}
\hline & Preplanned & Post Hoc & $P$ Value \\
\hline Inclusion/exclusion & & & \\
Subgroup studies & $3 / 17$ & $28 / 68$ & \\
RCTs & $10 / 49$ & & \\
Total & $13 / 66$ & $28 / 68$ & $.009^{\mathrm{a}}$ \\
Outcome & $13 / 84$ & $19 / 42$ & $.0005^{\mathrm{a}}$ \\
\hline a Fisher exact test & & &
\end{tabular}

eligibility: DWI to confirm the diagnosis before enrollment, the presence of edema on CT to exclude patients, and location of infarction within a specific vascular territory for either inclusion or exclusion (On-line Table 1).

Vessel occlusion by using angiography (CTA, MRA, or DSA) and TCD was commonly identified before enrollment in a trial. Of the 16 trials that selected according to vessel-occlusion status, 12 used imaging to identify a target for the intervention and the remaining 4 excluded patients with carotid occlusions on the basis of the futility of the intervention (On-line Table 1).

Perfusion deficit alone was used in only 2 studies to select patients for inclusion (Table 1), whereas 13 studies used reperfusion as an outcome criterion in 27 different efficacy analyses. There was considerable heterogeneity in the timing of the assessment of reperfusion (4 hours to 3 months), which was made using a variety of modalities (MRI perfusion, CTP, SPECT) (On-line Table 2).

Identification of the ischemic penumbra, as a means of either patient selection or outcome assessment, was common (Table 1). On-line Table 3 demonstrates the variety of ways in which penumbra has been defined. All except 1 study defined penumbra using MRI perfusion measures, such as mean transit time or timeto-maximum, to identify hypoperfused tissue that extended beyond the DWI lesion, and most used a threshold of 20\% mismatch to define ischemic penumbra to select patients. No trial using MRI perfusion resulted in a positive outcome. However, this threshold (20\% mismatch to define the ischemic penumbra) was used with success in an evaluation of tenecteplase by using CTP (On-line Table 3). ${ }^{5}$ In addition to trials of reperfusion therapies, several neuroprotective trials also used penumbral imaging for inclusion.

\section{Subgroup Analyses}

The comparison of the results of studies with preplanned imaging-defined subgroups or imaging-based outcomes compared with post hoc analyses can be seen in Table 2. There are significantly more positive results in studies using post hoc analyses. For instance, no preplanned subgroup analysis of mismatch eligibility criteria has demonstrated a positive effect of what otherwise had been a null trial by primary analysis, whereas 2 post hoc-defined subgroup analyses have demonstrated a positive effect (On-line Table 3): those patients with very large perfusion deficits and small DWI lesions ${ }^{6}$; and patients with a $20 \%$ mismatch profile only once images were properly coregistered. ${ }^{7}$ More generally, the definitions used for subgroup selection were less consistent than those used for trial eligibility criteria (On-line Table 4). Using the example of quantification of cerebral infarction, we found that definitions ranged from strata of absolute volumes to scoring systems such as the Alberta Stroke Programme Early CT Score. ${ }^{8}$

\section{DISCUSSION}

This review reinforces the framework outlined in the Acute Stroke Imaging Research Roadmap II. ${ }^{2}$ It identifies groupings of imaging biomarkers that have been used across RCTs, reflecting the individual needs of those trials. However, there is marked heterogeneity in the definition of these imaging biomarkers among trials, with a large number of post hoc subgroup analyses exploring further imaging biomarker definitions.

In general terms, the use of imaging biomarkers is intended to produce a more homogeneous population within a trial, with the hope of limiting patient selection to those for whom the intervention is most likely to be of benefit. This should allow smaller trials to be conducted over shorter periods. We identified 8 groupings of imaging biomarkers used in acute stroke trials: infarct volume, edema, diagnosis/territory of infarction, vessel status, ischemic penumbra, perfusion status, collaterals, and composite/other. All were used to evaluate trial eligibility or outcome assessment, or for subgroup analysis.

This review highlights the variability of imaging biomarker definitions and acquisitions in RCTs. Taking infarct volume as an example, one-third of the MCA territory as a threshold on noncontrast CT was the most consistent imaging biomarker used for trial eligibility. Less consistency was seen when infarct volume was used to assess outcome. Timing of measurements, technique used, and measurement technique were all highly variable among trials (On-line Table 2). When an imaging biomarker becomes more complex, the epitome being ischemic penumbra, even more heterogeneity exists (On-line Table 3).

The impact of the inconsistent measurement of imaging biomarkers is important. For example, if infarct volume is used as an outcome assessment, edema and atrophy can affect the infarct volume at different times. ${ }^{9}$ Within the same patients the blinded adjudication of FLAIR and T2 images by neuroradiologists results in different infarct volume estimates and discrepant interrater agreements. ${ }^{10}$ The reliability and repeatability of a biomarker in defining a pathologic process will be affected by the contrast-tonoise ratio afforded by the imaging technique. ${ }^{11}$ For example, DWI has a greater contrast-to-noise ratio than T2-weighted MR imaging, CT, and CTP, allowing a clearer definition of the extent of a lesion, ${ }^{11,12}$ thus reducing measurement error and improving interrater agreement. ${ }^{13}$ Even when there is excellent interrater agreement among neuroradiologists, substantial measurement errors can still exist that affect sample-size calculation for a RCT, particularly when the infarct volumes are small or moderate. ${ }^{14}$ Automated approaches to volume measurement offer the prospect of limiting human measurement error but introduce challenges of their own in the accommodation of thresholds that vary among individuals and that are also influenced by timing from stroke onset. ${ }^{14}$

The relationship between patients, imaging biomarkers, treatments, and eventual clinical outcomes is complex. Failure to accurately understand these relationships and, thus, selecting an inappropriate biomarker have contributed to the criticisms of recent RCTs. Defining a treatment-responsive group by using an 
imaging biomarker is an entirely different task to selecting those who are destined to do well, independent of treatment, as was seen in the Mechanical Retrieval and Recanalization of Stroke Clots Using Embolectomy study. ${ }^{15,16}$ The only positive RCT of intravenous thrombolysis used within 3 hours of stroke onset is not included in this review because patients were not excluded by infarct volume criterion. ${ }^{17}$ Of note, post hoc analysis demonstrated that infarct volume was not a treatment modifier within 3 hours. ${ }^{18}$ The optimal threshold of infarct volume used to exclude patients from trial enrollment remains unclear. ${ }^{6,19}$

Several prospective observational studies have explored alternate definitions of the ischemic core using DWI to select patients for endovascular therapy (Diffusion and Perfusion Imaging Evaluation for Understanding Stroke Evolution Study 2 used a threshold of $>70 \mathrm{~mL}$ on DWI to exclude patients). ${ }^{19-22}$ These data suggest that patients selected by using lower infarct volume thresholds than used previously have a greater capacity to benefit from endovascular treatment. This promising approach from observational studies now needs to be translated into RCTs.

Expected treatment effects on a biomarker used as an imaging outcome and its relationship with clinical outcomes also need to be understood when designing a RCT. For example, the recanalization rate with intravenous IPA in the Interventional Management of Stroke III trial was twice what was predicted, and the highly significant increase in revascularization was not associated with improvement in clinical outcomes. ${ }^{23}$

The Acute Stroke Imaging Research Roadmap II outlines a framework for use within a RCT, advocating the use of a consistent Treatment-Relevant Acute Imaging Target within all arms. This would allow secondary analyses to address the additional value of imaging while the primary focus remains on the therapeutic intervention. This area deserves further methodologic consideration; this review highlights the large number of subgroup analyses, typically post hoc, performed using data from the RCTs. We found an excess of positive post hoc subgroup analyses, suggesting that any apparently useful subgroups should be viewed with caution, given the risk of publication bias. ${ }^{1,24}$ This may give a false sense of promise from apparently successful post hoc attempts to stratify patients (Table 2 ), meaning that these positive results should be used only for hypothesis generation and validation in separate patient research cohorts, and before widespread clinical adoption. ${ }^{25}$

Successful meta-analyses of pooled data of RCTs of intravenous thrombolysis and hemicraniectomy have contributed to their wide adoption into national stroke clinical guidelines and practice. ${ }^{15,16}$ The inconsistent use of imaging biomarkers highlighted in this review markedly hampers such meta-analyses. For instance, it would be inappropriate to combine cohorts in whom mismatch was defined using different modalities and thresholds. It is equally challenging to interpret results from individual studies in which different imaging modalities are used to define enrollment criteria: for instance, the Mechanical Retrieval and Recanalization of Stroke Clots Using Embolectomy study used either MRI or CT to assess the favorability of the penumbral pattern. ${ }^{15}$ The advantage of this approach is to maximize the number of sites that may take part in a RCT, hopefully reducing study duration and broadening the generalizability of the results. How- ever, unless the equivalence of the biomarkers is robustly established, the reliability of data interpretation is potentially compromised. The Acute Stroke Imaging Research Roadmap II begins to address these difficulties with the example of the clarification of revascularization status and associated concepts. ${ }^{2,26}$

There remains an unmet need for a robust pathway for stroke imaging biomarker development from the preclinical studies through translational and observational studies ready for use in RCTs and clinical settings. This pathway needs to accommodate the possibility that the imaging that drives scientific discovery may or may not be available for clinical use in acute stroke. For example, diffusion MRI is a widely used and validated method for identifying core infarct in animal model studies and has been used in several RCTs. On the other hand, perfusion CT has almost no preclinical validation but is commonly used in RCTs. The relationship between how and whether different imaging modalities measure the same pathophysiology must be established. Existing initiatives developed from expert consensus may expedite this process and ensure a more homogeneous use of biomarkers in RCTs. ${ }^{27,28}$

\section{CONCLUSIONS}

Imaging biomarkers offer the opportunity to refine the trial cohort by minimizing participant variation, to decrease sample size, and to personalize treatment approaches for those who stand to benefit most. However, within imaging modalities, there has been little consistency among stroke trials. Greater effort to prospectively use consistent imaging biomarkers should help improve the development of novel treatment strategies in acute stroke and improve comparison between studies.

\section{ACKNOWLEDGMENTS}

We wish to acknowledge the facilities provided by the Oxford Acute Vascular Imaging Centre.

Disclosures: George W.J. Harston—RELATED: Grant: research grants via the University of Oxford, ${ }^{*}$ Comments: Dunhill Medical Trust, National Institute for Health Research Oxford Biomedical Research Centre, Centre of Excellence for Personalized Healthcare, funded by the Wellcome Trust and Engineering and Physical Sciences Research Council. Neil Rane-UNRELATED: Travel/Accommodations/Meeting Expenses Unrelated to Activities Listed: received travel grants and participated in funded meetings from Codman Neurovascular, MicroVention, Stryker, Covidien; Other: current fellowship position partly funded by Codman Neurovascular, not institutionally organized by the author; received scholarship funding for current position from HCA International Foundation, United Kingdom (private hospital group). Please also see departmental disclosures of the first author. Sivarajan Thandeswaran-RELATED: Grant: National Institute for Health Research Oxford Biomedical Research Centre, * Dunhill Medical Trust, * Centre of Excellence for personalized health care funded by the Wellcome Trust and the Engineering and Physical Sciences Research Council.* Fintan Sheerin—RELATED: Grant: See lead author's disclosures for funding/grant details*; UNRELATED: Expert Testimony: second opinion on clinical scans provided for a patient challenging a decision by their health insurance company. I do not currently do medicolegal work; Payment for Lectures (including service on Speakers Bureaus): Oxford MRI and Oxford Neuroradiology courses, Comments: honorarium for educational lectures; Travel/Accommodations/Meeting Expenses Unrelated to Activities Listed: study expenses: Oxford University Hospitals study funds paid for British Society of Neuroradiologists conference fee 2013. *Money paid to the institution.

\section{REFERENCES}

1. Wardlaw JM. Surrogate outcomes: a cautionary note. Stroke 2009; 40:1029-31 
2. Wintermark M, Albers GW, Broderick JP, et al. Acute Stroke Imaging Research Roadmap II. Stroke 2013;44:2628-39

3. Wintermark M, Sanelli PC, Albers GW, et al. Imaging recommendations for acute stroke and transient ischemic attack patients: a joint statement by the American Society of Neuroradiology, the American College of Radiology, and the Society of NeuroInterventional Surgery. AJNR Am J Neuroradiol 2013;34:E117-27

4. Vahedi K, Vicaut E, Mateo J, et al. Sequential-design, multicenter, randomized, controlled trial of early decompressive craniectomy in malignant middle cerebral artery infarction (DECIMAL Trial). Stroke 2007;38:2506-17

5. Parsons M, Spratt N, Bivard A, et al. A randomized trial of tenecteplase versus alteplase for acute ischemic stroke. $N$ Engl J Med 2012;366:1099-107

6. Parsons MW, Christensen S, McElduff P, et al. Pretreatment diffusion- and perfusion-MR lesion volumes have a crucial influence on clinical response to stroke thrombolysis. J Cereb Blood Flow Metab 2010;30:1214-25

7. Nagakane Y, Christensen S, Brekenfeld C, et al. EPITHET: positive result after reanalysis using baseline diffusion-weighted imaging/ perfusion-weighted imaging co-registration. Stroke 2011;42:59-64

8. Barber PA, Demchuk AM, Zhang J, et al. Validity and reliability of a quantitative computed tomography score in predicting outcome of hyperacute stroke before thrombolytic therapy: ASPECTS Study Group-Alberta Stroke Programme Early CT Score. Lancet 2000; 355:1670-74

9. Inoue M, Mlynash M, Christensen S, et al. Early diffusion-weighted imaging reversal after endovascular reperfusion is typically transient in patients imaged 3 to 6 hours after onset. Stroke 2014; 45:1024-28

10. Neumann $\mathrm{AB}$, Jonsdottir $\mathrm{KY}$, Mouridsen $\mathrm{K}$, et al. Interrater agreement for final infarct MRI lesion delineation. Stroke 2009;40: 3768-71

11. González RG, Schaefer PW, Buonanno FS, et al. Diffusion-weighted MR imaging: diagnostic accuracy in patients imaged within 6 hours of stroke symptom onset. Radiology 1999;210:155-62

12. González RG. Low signal, high noise and large uncertainty make CT perfusion unsuitable for acute ischemic stroke patient selection for endovascular therapy. J Neurointerv Surg 2012;4:242-45

13. Campbell BC, Tu HT, Christensen S, et al. Assessing response to stroke thrombolysis: validation of 24-hour multimodal magnetic resonance imaging. Arch Neurol 2012;69:46-50

14. Ay H, Arsava EM, Vangel M, et al. Interexaminer difference in in- farct volume measurements on MRI: a source of variance in stroke research. Stroke 2008;39:1171-76

15. Kidwell CS, Jahan R, Gornbein J, et al. A trial of imaging selection and endovascular treatment for ischemic stroke. $N$ Engl J Med 2013;368:914-23

16. Powers WJ. Intravenous thrombolysis of basilar artery thrombosis. Ann Neurol 2014;75:456-57

17. National Institute of Neurological Disorders and Stroke rt-PA Stroke Study Group. Tissue plasminogen activator for acute ischemic stroke. N Engl J Med 1995;333:1581-87

18. Patel SC, Levine SR, Tilley BC, et al. Lack of clinical significance of early ischemic changes on computed tomography in acute stroke. JAMA 2001;286:2830-38

19. Lansberg MG, Straka M, Kemp S, et al. MRI profile and response to endovascular reperfusion after stroke (DEFUSE 2): a prospective cohort study. Lancet Neurol 2012;11:860-67

20. Simonsen CZ, Sørensen LH, Karabegovic S, et al. MRI before intraarterial therapy in ischemic stroke: feasibility, impact, and safety. J Cereb Blood Flow Metab 2014;34:1076-81

21. Wisco D, Uchino K, Saqqur M, et al. Addition of hyperacute MRI aids in patient selection, decreasing the use of endovascular stroke therapy. Stroke 2014;45:467-72

22. Yoo AJ, Verduzco LA, Schaefer PW, et al. MRI-based selection for intra-arterial stroke therapy: value of pretreatment diffusionweighted imaging lesion volume in selecting patients with acute stroke who will benefit from early recanalization. Stroke 2009; 40:2046-54

23. Broderick JP, Palesch YY, Demchuk AM, et al. Endovascular therapy after intravenous t-PA versus t-PA alone for stroke. $N$ Engl J Med 2013;368:893-903

24. Tzoulaki I, Siontis KC, Evangelou E, et al. Bias in associations of emerging biomarkers with cardiovascular disease. JAMA Intern Med 2013;173:664-71

25. Rothwell PM. Treating individuals 2: subgroup analysis in randomised controlled trials: importance, indications, and interpretation. Lancet 2005;365:176-86

26. Tomsick T. TIMI, TIBI, TICI: I came, I saw, I got confused. AJNR Am J Neuroradiol 2007;28:382-84

27. European Society of Radiology. White paper on imaging biomarkers. Insights Imaging 2010;1:42-45

28. Kessler LG, Barnhart HX, Buckler AJ, et al. The emerging science of quantitative imaging biomarkers terminology and definitions for scientific studies and regulatory submissions. Stat Methods Med Res 2015;24:9-26 